\title{
Numerical Momentum Tracking for a Hall Thruster Plume
}

\author{
Iain D. Boyd ${ }^{\mathrm{a}}$ \\ University of Michigan, Ann Arbor, Michigan 48109, USA \\ Mark W. Crofton ${ }^{\mathrm{b}}$ \\ The Aerospace Corporation, El Segundo, California 90245, USA
}

\begin{abstract}
Numerical simulation techniques were used to track the evolution of species-specific momentum throughout a 5-kW class Hall thruster plume, with inclusion of magnetic field effects. All significant particle interactions were included in the model, and specific emphasis was placed on the dependence of velocity distribution functions and total plume momentum on the density of background xenon gas. To obtain the results, the coordinate-dependent influence of plume parameters such as ion flux density, neutral density, electron temperature and plasma potential was included in the calculations. Plasma potential increased significantly when the magnetic field was present. Results show that significant plume interactions occur, with initial ion beam momentum becoming substantially distributed between ion and neutral populations as the ion beam is attenuated, and increasing total momentum flux across a thruster-centric hemispherical surface at increasing downstream distance. Velocity distribution functions continue to change with propagation distance beyond $1 \mathrm{~m}$ and exhibit angular and pressure dependence. Collisionbased reduction of average ion momentum occurs in the plume as average neutral momentum increases. Integrated momentum flux variation with background pressure is obtained, due mainly to slow ions produced by charge exchange and electron impact ionization. These ions gain much more velocity than beam ions for an equivalent potential drop, but their trajectories are generally more radial than axial. Axial momentum contributions are sensitive to details of the plume potential map, with individual ions contributing negatively or positively depending on birthplace and resulting trajectory. The predicted increase of plume momentum with rising background gas density has similarities to the result of an earlier 1-D analytical study, although of lower magnitude and no longer monotonic.
\end{abstract}

\section{Introduction}

$\mathrm{H}$ igh brightness, broad beam xenon ion sources operated in a ground-based environment generate a background of neutral xenon gas, due to finite pumping speed of the vacuum facility. Penetration of the neutrals into the ion beam plasma and interaction with its constituents may modify plasma potential, density, temperature, velocity and trajectory characteristics. Ultimately, thruster performance figures can be affected. This "facility effect" and others have produced numerous unexplained results over the years. ${ }^{1}$

Propellant atoms are ionized efficiently in modern ion propulsion devices, the majority of which rely on xenon propellant. While the ion flux in the plume is much higher than neutral flux, neutral density normally dominates over plasma density. During ground operation, the density of background neutrals is established by thruster flow rate and pumping speed of the facility. In the present study we focus on the effects of neutral background gas density and its interaction with beam ions through charge exchange (CEX) reactions. In addition to alteration of the plasma density profile a principal macroscopic effect of the interaction is modification of performance metrics of an operating propulsion device. Several thrust modification mechanisms exist that are associated with neutrals. Neutrals that diffuse into plume regions near a thruster exit plane may undergo ionization and acceleration. This

${ }^{\mathrm{a}}$ Professor, Department of Aerospace Engineering, Fellow AIAA.

${ }^{\mathrm{b}}$ Senior Scientist, Propulsion Science Department, M2-341, AIAA Senior Member. 
process produces a more direct augmentation of thrust than charge exchange. Ion-neutral collisions impede neutrals from diffusing into the core of the ion beam and reduce the average energy of plume ions. A thrust enhancement will result if there is subsequent axial momentum gain for the just-formed slow xenon ion; the sum of beam and charge exchange ion pair momentum will exceed that of a beam ion that did not charge exchange.

Xenon neutral density and its spatial and temporal variation is a key determinant of Hall thruster behavior. Thruster stability, optimal set-points, and performance characteristics are all influenced by both internal and external density. Neutrals that diffuse into plume regions near the thruster exit plane and undergo ionization and acceleration, or momentum exchange with ions, add to spacecraft integration issues at high angles with respect to the thrust axis. These effects tend to be larger for Hall thrusters than ion engines, of the same class.

Xenon neutrals from the background gas diffuse into the plume of a thrust-generating ion beam and undergo reactions of several types. The most important for thrust generation are electron impact ionization and charge exchange ionization. Charge exchange reactions with approximately zero momentum transfer, where a fast singly charged beam ion and slow neutral atom are efficiently converted into a slow ion and a fast neutral atom according to $\mathrm{Xe}^{+}$(fast) $+\mathrm{Xe}$ (slow) $\rightarrow \mathrm{Xe}$ (fast) $+\mathrm{Xe}^{+}$(slow), are of the most significance. Ion-neutral and neutral-neutral collisions that produce scattering at various angles are also important, and their effects on plume divergence of Hall Effect Thrusters (HETs) have been studied before. ${ }^{2}$

Recent development and application of a 1-D analytical model ${ }^{3}$ to calculate neutral density in the operating xenon HET plume predicted neutral depletion would occur in the core of the ion beam. Analysis of ion-neutral charge exchange reactions concluded that thrust augmentation would occur due to collision-based reduction of average ion momentum with concurrent increases of average neutral momentum, and the on-going acceleration of ion populations in the local electric field to increase their momentum. Downshifting the average ion energy by flowing it into neutral energy augments plume momentum, because kinetic energy depends on the square of velocity whereas momentum is proportional to velocity, and a given energy change produces a greater velocity increment for low energy populations. The result is increased plume momentum that may be registered as thrust exerted by the HET.

Direct Simulation Monte Carlo (DSMC) techniques are used here for the first time to track the evolution of species-specific and total plume momentum throughout a Hall thruster plume. Detailed calculations were performed to determine velocity and density distribution functions for the population of ions and neutrals throughout the HET plume. A DSMC approach was used to model the collisions of the ions and neutrals, with Particle-in-Cell (PIC) modeling of ion transport in the plume electric field. All significant particle interactions were included in the model, and specific emphasis was placed on the dependence of velocity distribution functions and total plume momentum on the density of background xenon gas. To obtain the results, the coordinate-dependent influence of plume parameters such as ion flux density, neutral density, electron temperature and plasma potential was included in the calculations.

Ion velocity distribution functions have been measured and modeled previously for internal environments and near field plumes of lower power Hall thrusters, exploring time-dependent variation of the electric field and VDF. ${ }^{4}$ Neutral measurement and modeling studies have shown that the VDF evolves in the flow field, with ion-neutral and neutral-neutral interactions influencing the profile. ${ }^{4,5}$

\section{Hall Thruster Plume Model}

The xenon plasma plume of a Hall thruster is composed of beam ions with velocities on the order of $16 \mathrm{~km} / \mathrm{s}$, low energy charge exchange ions, neutral atoms, and electrons. The total number density in the thruster is of the order of $10^{18} \mathrm{~m}^{-3}$, placing the plasma in the rarefied flow regime. Computational analysis of Hall thruster plumes is regularly performed using a hybrid particle-fluid formulation. ${ }^{6,7}$ The direct simulation Monte Carlo (DSMC) method $^{8}$ models the collisions of the heavy particles (ions and atoms). The Particle In Cell (PIC) method ${ }^{9}$ models the transport of the ions in electric fields. Overall, a hybrid approach is employed in which the electrons are modeled using a fluid description. In the present study, we employ a detailed treatment of the electron fluid by simultaneous solution of its three conservation equations. ${ }^{10}$

\section{Plasma Dynamics}

The most successful Hall thruster plume models are based on a hybrid approach in which heavy species are modeled using particles and the electrons modeled as a fluid. In the present study, a detailed fluid electron model is employed that uses all three electron conservation equations. The model has been described in detail elsewhere and applied to the near field of a $200 \mathrm{~W}$ Hall thruster operated in a test chamber. ${ }^{10}$

The electron continuity equation is ${ }^{11}$ 


$$
\frac{\partial}{\partial t}\left(n_{e}\right)+\nabla \cdot\left(n_{e} \bar{V}_{e}\right)=n_{e} n_{a} C_{i}
$$

where $\bar{V}_{e}$ is the electron velocity vector, $n_{a}$ is the atomic number density, and $C_{i}$ is the ionization rate coefficient. Assuming steady flow, this equation is transformed into a Poisson equation by introducing a stream function $\psi$ using

$$
n_{e} \bar{v}_{e}=\nabla \psi
$$

such that

$$
\nabla^{2} \psi=n_{e} n_{a} C_{i}
$$

for which numerical solutions are obtained using the standard Alternating Direction Implicit (ADI) method. The spatial distribution of the ion particles gives the electron number density, $n_{e}$, under the assumption of charge neutrality. This allows the electron velocity vector to be determined through solution of (3). In the present study, the xenon ionization rate coefficient is set to zero due to the low temperatures involved.

Assuming steady state, the electron momentum equation is given by ${ }^{6}$

$$
m_{e} n_{e}\left(\bar{V}_{e} \cdot \nabla\right) \bar{V}_{e}=-e n_{e}\left(\bar{E}+\bar{V}_{e} \times \bar{B}\right)-\nabla p_{e}+\bar{R}
$$

where $m_{e}$ is the mass of an electron, $\bar{E}$ is the electric field, $\bar{B}$ is the magnetic field, $p_{e}$ is the electron pressure, and $\bar{R}$ is the friction term. It is further assumed that the electrons behave as a perfect gas ( $p_{e}=n_{e} k T_{e}$ ), and that the friction term is given by:

$$
\bar{R}=\frac{e n_{e} \bar{j}}{}
$$

where $\bar{j}$ is the current density, and is the electrical conductivity.

Neglecting the inertial term on the left hand side of (4), and introducing the plasma potential $-\nabla \phi=\bar{E}$, a generalized Ohm's law is obtained:

$$
\nabla \cdot \bar{j}=\nabla \cdot \sigma\left[-\nabla \varphi+\bar{V}_{e} \times \bar{B}+\frac{1}{e n_{e}} \nabla\left(n_{e} k T_{e}\right)\right]
$$


Assuming that the magnetic field of a Hall thruster is mainly radial, so only $B_{r}$ need be considered, Eq. (6) can be solved provided that an estimate of the azimuthal electron velocity is obtained. A linear combination of collisional and collisionless results is employed ${ }^{12}$

$$
V_{e}=\left(\begin{array}{ll}
1 & w
\end{array}\right) V_{d r i f t}+w \quad \frac{e}{m_{e} e} V_{e z} B_{r} \div
$$

where $w=n / n_{\max }$ and $n_{\max }$ is the maximum number density.

For given $n_{e}, \bar{v}_{e}$, and $T_{e}$, the charge continuity condition:

$$
\nabla \cdot \bar{j}=0
$$

is then solved to obtain the plasma potential. This equation is written as a Laplace equation with weak source terms and is again solved using an Alternating Direction Implicit (ADI) scheme.

The steady state electron energy equation is given by ${ }^{11}$

$$
\frac{3}{2} n_{e}\left(\bar{v}_{e} \cdot \nabla\right) k T_{e}+p_{e} \nabla \cdot \bar{v}_{e}=\nabla \cdot \kappa_{e} \nabla T_{e}+\bar{j} \cdot \bar{E}-3 \frac{m_{e}}{m_{i}} v_{e} n_{e} k\left(T_{e}-T_{H}\right)-n_{e} n_{a} C_{i} \varepsilon_{i}
$$

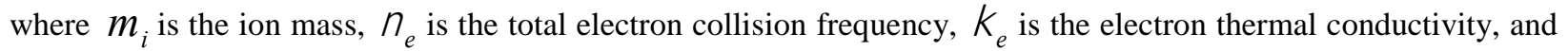
$T_{H}$ is the heavy particle temperature. Again assuming a steady state, and dividing by the thermal conductivity:

$$
\nabla^{2} T_{e}=-\nabla \ln \left(\kappa_{e}\right) \cdot \nabla T_{e}+\frac{1}{\kappa_{e}}\left(-\bar{j} \cdot \bar{E}+\frac{3}{2} n_{e}\left(\bar{v}_{e} \cdot \nabla\right) k T_{e}+p_{e} \nabla \cdot \bar{v}_{e}+3 \frac{m_{e}}{m_{i}} v_{e} n_{e} k\left(T_{e}-T_{H}\right)+n_{e} n_{a} C_{i} \varepsilon_{i}\right)
$$

where $\bar{j}$ is obtained from (6) after the plasma potential is calculated. Equation (10) is again a Laplace equation with weak source terms that is solved using the ADI approach.

Finally, the electron transport coefficients are evaluated from their basic definitions ${ }^{11}$

$$
\begin{aligned}
& =\frac{e^{2} n_{e}}{m_{e} e} \\
& e=\frac{2.4}{1+\frac{e i}{\sqrt{2}}} \frac{k^{2} n_{e} T_{e}}{m_{e} e}
\end{aligned}
$$


where $e_{e}={ }_{e i}+e_{e n}, \quad e_{e i}$ is the ion-electron collision frequency, $e_{e n}$ is the neutral-electron collision frequency, and these frequencies are evaluated for the xenon system using cross sections provided in [6]. Note that, for each time step, the numerical scheme iterates several times through the solution of (7) and (10) due to the coupling between and $T_{e}$.

\section{Collision Dynamics}

The DSMC method uses particles to simulate collision effects in rarefied gas flows by collecting groups of particles into cells which have sizes of the order of a mean free path. Pairs of these particles are then selected at random and a collision probability is evaluated that is proportional to the product of the relative velocity and collision cross section for each pair. The probability is compared with a random number to determine if that collision occurs. If so, some form of collision dynamics is performed to alter the properties of the colliding particles.

There are two basic classes of collisions that are important in the Hall thruster plumes: (1) elastic (momentum exchange); and (2) charge exchange. Elastic collisions involve only exchange of momentum between the participating particles. For the systems of interest here, this may involve atom-atom or atom-ion collisions. For atom-atom collisions, the Variable Hard Sphere (VHS) [3] collision model is employed. For xenon, the collision cross section is

$$
{ }_{E L}(X e, X e)=\frac{2.12 \quad 10^{18}}{g^{2}}
$$

where the units are $\mathrm{m}^{2}, \mathrm{~g}$ is the relative velocity, and $\quad=0.12$ is related to the viscosity temperature exponent for xenon.

Both atom-ion momentum and charge exchange processes are modeled using the same cross section that was developed by Boyd and Dressler ${ }^{7}$ based on a combination of theory and experiment:

$$
{ }_{C E X}\left(X e, X e^{+}\right)=\left(13.6 \log _{10}()+87.3\right) 10^{20}
$$

where $=\frac{1}{2} m^{*} g^{2}$ and is measured in $\mathrm{eV}$, and $m^{*}$ is the reduced mass. Reference 7 also recommends that charge exchange cross sections for the interaction where a doubly charged ion captures two electrons from an atom are a factor of two lower than the values for the singly charged ions at corresponding energies. Charge-exchange collisions are simulated using the differential cross sections developed in Ref. 7. Momentum exchange collisions assume either the same differential scattering cross sections, or isotropic scattering.

\section{Boundary Conditions}

For the computations of Hall thruster plumes, boundary conditions must be specified at several locations: (1) at the thruster exit; (2) at the cathode exit; (3) along the outer edges of the computational domain; and (4) along all solid surfaces in the computational domain.

Several macroscopic properties of the plasma exiting the thruster are required for the computations. For the electrons, their current, temperature, and the plasma potential must be specified. For each of the heavy species (Xe, $\left.\mathrm{Xe}^{+}, \mathrm{Xe}^{2+}\right)$ we require the number density, velocity, and temperature. In the real device, these properties vary radially across the exit plane.

The approach to determining these properties involves a mixture of analysis and estimation. The basic performance parameters of mass flow rate, thrust, and total ion current are assumed known. The neutrals are assumed to exit the thruster at the sonic speed corresponding to some assumed value for their temperature. Finally, divergence angles for the lower and upper edges of the exit channel must be assumed. Combining all this information then allows all species densities and the velocities to be determined. Determination of the properties of multiple charge states, for example $\mathrm{Xe}^{2+}$ is considered in the present study, requires knowledge of the current fraction of that state. The major plasma properties at the thruster exit are summarized in Table I. 
In the electron fluid model, the external cathode of the Hall thruster must be modeled. While the actual cathode provides essentially a point source of electrons that therefore involves a three dimensional flow, in the present study it is modeled within the axially symmetric framework of the code. This is not a bad assumption given the high mobility of the electrons that rapidly form a symmetric flow field. The boundary conditions required for the electrons at the exit of the cathode are the electron current, the plasma potential, and the electron temperature.

Both fluid and particle boundary conditions are required at the outer edges of the computational domain. The electron fluid conditions employ either Dirichlet (fixed value) or Neumann (fixed gradient) boundary conditions. For the simulation of a spacecraft plume in space, these conditions are difficult to determine. In the present case, the gradients of electron current normal to the domain edges are set to zero, and the plasma potential on all domain boundaries is set to $3 \mathrm{~V}$. The electron temperature along the outer edges of the domain is usually set to $1.2 \mathrm{eV}$. The heavy particle boundary condition is to simply remove from the computation any particle crossing any domain edge.

The solid wall surfaces of the Hall thruster are also included in the computation. Along these walls, a fixed value $(2 \mathrm{eV})$ of the electron temperature is usually employed. The front face of the thruster is sprayed with di-electric material and so a condition of zero current is employed to calculate the plasma potential. In terms of heavy particles, any ions colliding with the walls are neutralized. Both atoms and neutralized ions are scattered back into the flow field from the surface of the thruster wall assuming diffuse reflection at a temperature of $500 \mathrm{~K}$.

The simulations include the finite background gas pressure of the experimental facility. This gas is initialized to a given pressure and density at a temperature of $300 \mathrm{~K}$. The nominal facility pressure of $1.1 \times 10^{-5}$ torr $\left(4 \times 10^{17} \mathrm{~m}^{-3}\right.$ is the corresponding density) is designated $1 \mathrm{X}$. In this nomenclature $0 \mathrm{X}$ refers to space conditions and $2 \mathrm{X}$ refers to twice the nominal operational facility pressure. As the thruster plasma plume expands into the background gas, the collisional interactions are simulated. Total flow rate for the simulation was $15.9 \mathrm{mg} / \mathrm{s}$ and thruster input power was $4.5 \mathrm{~kW}(14.9 \mathrm{~A}$ at $300 \mathrm{~V})$.

Magnetic and electric fields, and electron impact ionization of neutral atoms in the plume were included in the simulation. Initial plume divergence was assumed as a boundary condition in each case, based on measurements of ion flux at $1 \mathrm{~m}$ for the $1 \mathrm{X}$ operating condition. Plasma potential equal to $3 \mathrm{~V}$ was imposed as downstream boundary condition for most of the simulations. Transient effects, such as oscillations, were not included.

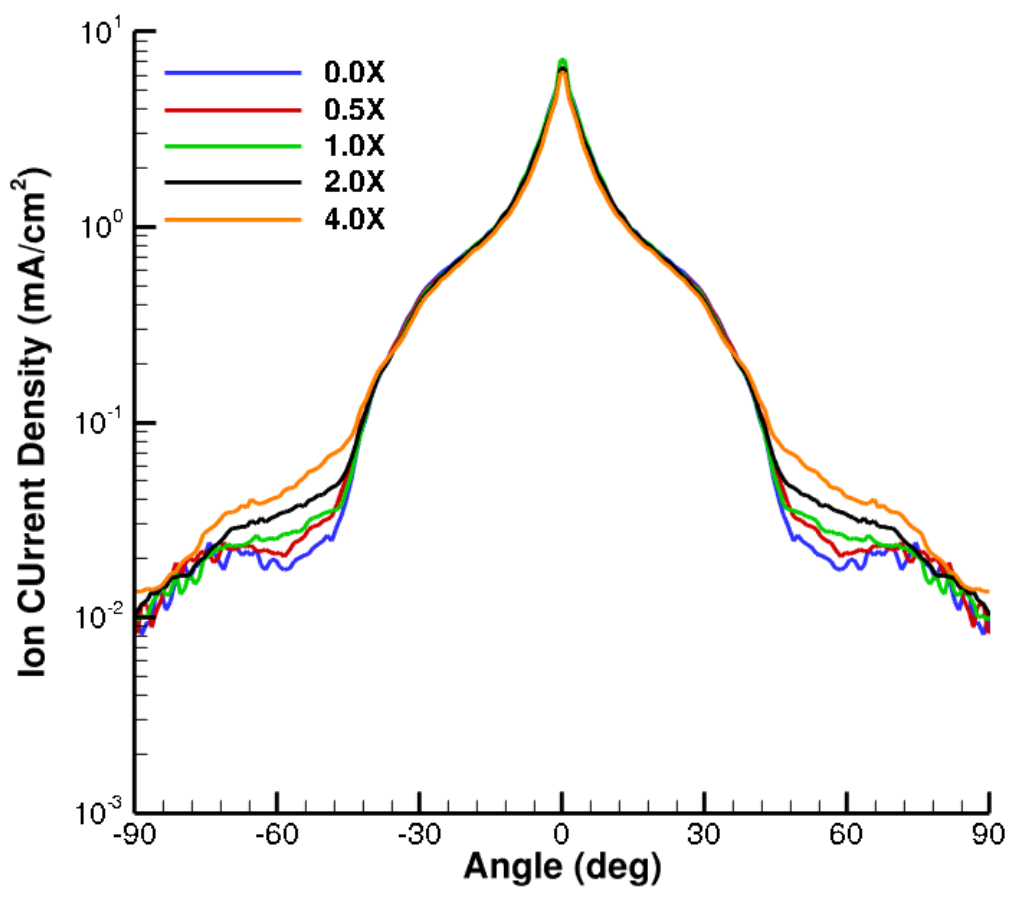

Figure 1. Angular dependence of plume ion flux, $1 \mathrm{~m}$ from thruster exit, at various background pressures (1X refers to facility pressure at the nominal $4.5 \mathrm{~kW}$ operating point). 
Table 1. Properties used in the simulation at the exit of the Hall thruster.

\begin{tabular}{ll}
\hline Inner Diameter $(\mathrm{cm})$ & 10 \\
\hline Outer Diameter $(\mathrm{cm})$ & 14 \\
\hline Plasma Density $\left(\mathrm{m}^{-3}\right)$ & $3.7 \times 10^{17}$ \\
\hline Neutral Density $\left(\mathrm{m}^{-3}\right)$ & $7.8 \times 10^{18}$ \\
\hline Ion Velocity $(\mathrm{m} / \mathrm{s})$ & 18,000 \\
\hline Neutral Velocity $(\mathrm{m} / \mathrm{s})$ & 280 \\
\hline Ion Temperature $(\mathrm{eV})$ & 2.0 \\
\hline Neutral Temperature $(\mathrm{K})$ & 750 \\
\hline Electron Temperature $(\mathrm{eV})$ & 8.0 \\
\hline Plasma Potential $(\mathrm{V})$ & 79 \\
\hline
\end{tabular}

\section{Results and Discussion}

Angular dependence of the plume ion flux at 1-m distance is plotted in Figure 1 as a function of xenon background pressure. The core of the ion beam is uninfluenced by background pressure, but pressure dependence is substantial outside of $\theta= \pm 40$ degrees, where $\theta$ is the off-axis angle. From 40 to 75 degrees off-axis there is 2 to 3 times greater ion current density at $4 \mathrm{X}$ pressure compared to $1 \mathrm{X}$, and the $1 \mathrm{X}$ to $4 \mathrm{X}$ flux progression may be nonlinear (i.e., $\Delta \mathrm{J} / \Delta \mathrm{P}$, where $\mathrm{J}$ is current density and $\mathrm{P}$ is pressure, is larger at low pressure). The ion current density variation is attributed to CEX ions formed predominately at angles less than 40 degrees. They migrate preferentially in the radial direction, but few have reached $\theta>75$ degrees, at $1 \mathrm{~m}$ distance. These simulation results compare well with available experimental data. ${ }^{13}$

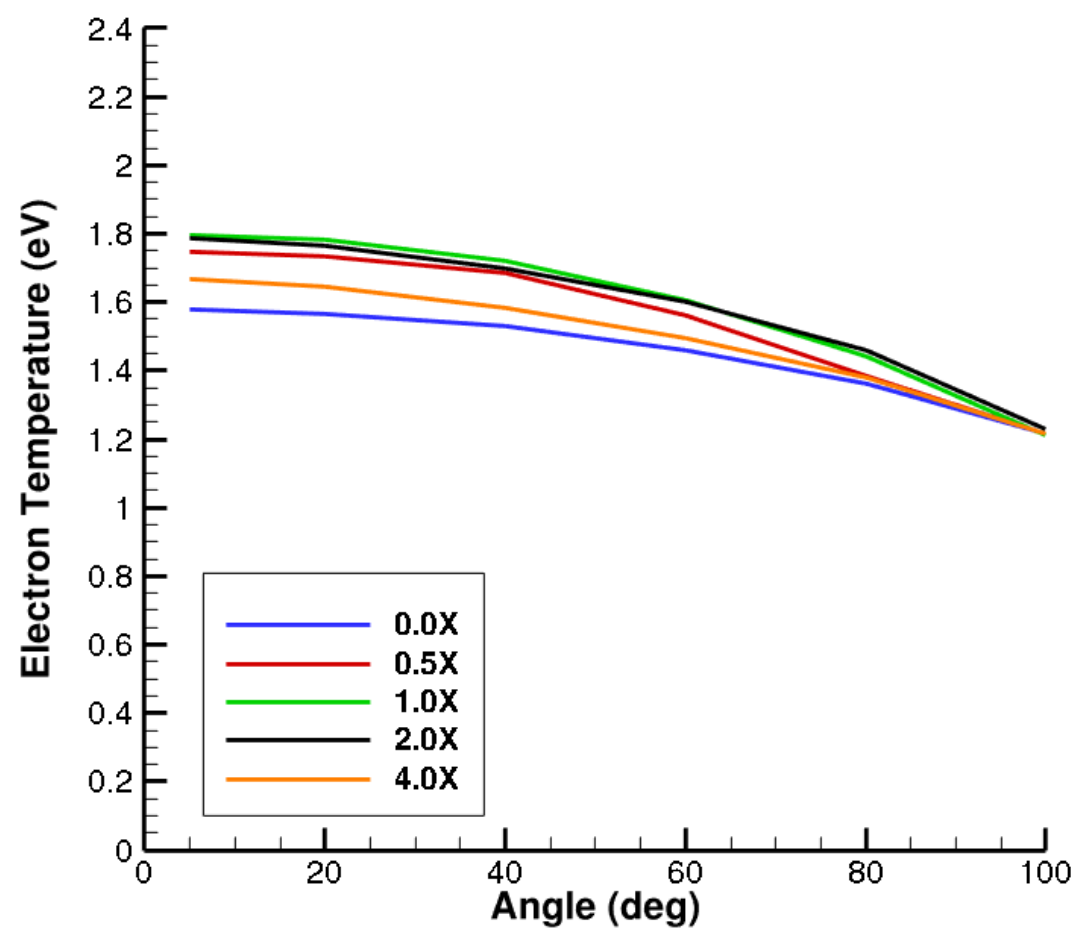

Figure 2. Angular dependence of plume electron temperature, $1 \mathrm{~m}$ from thruster exit, at various background pressures. 


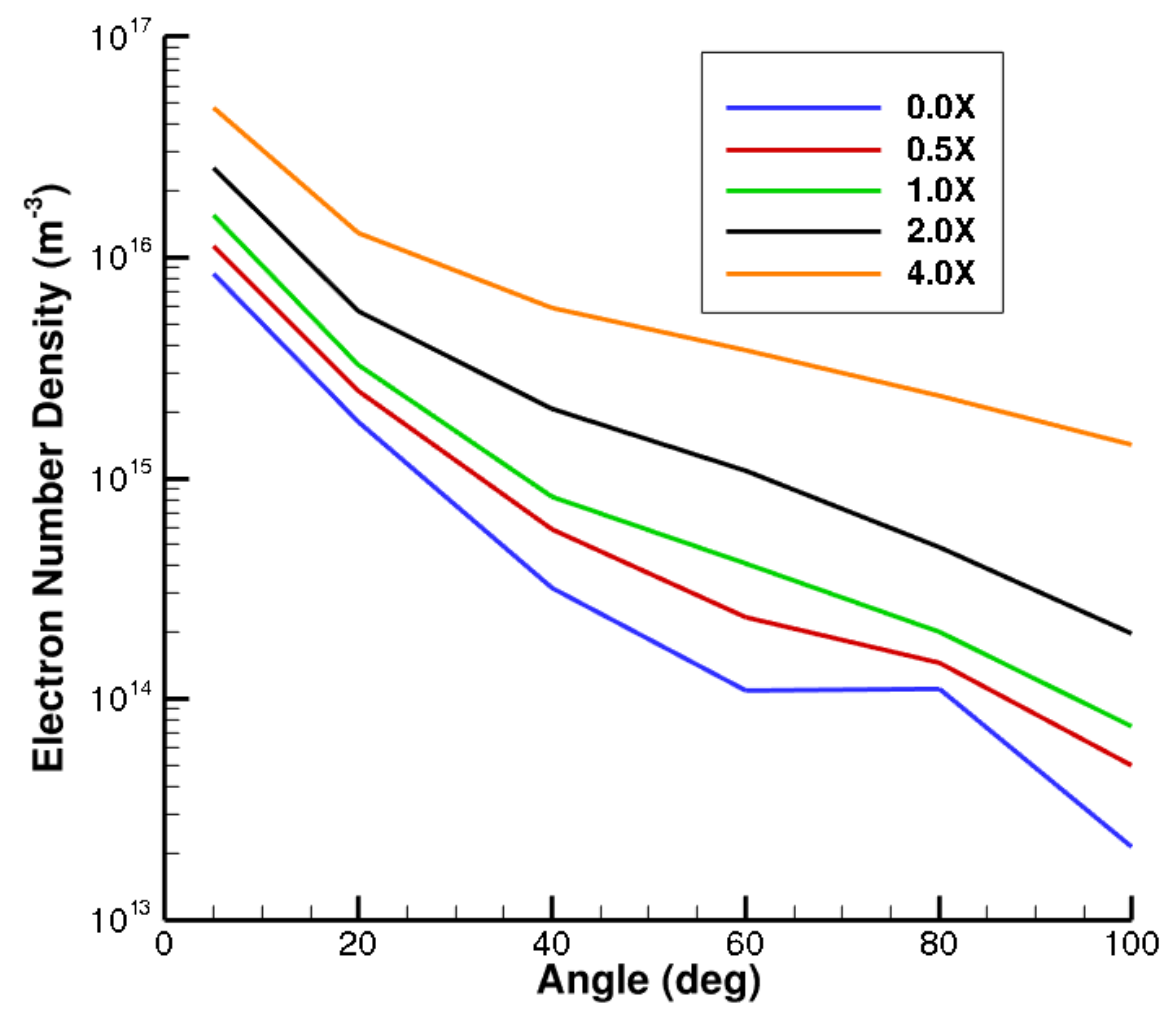

Figure 3. Angular dependence of electron density, $1 \mathrm{~m}$ from thruster exit, at various background pressures.

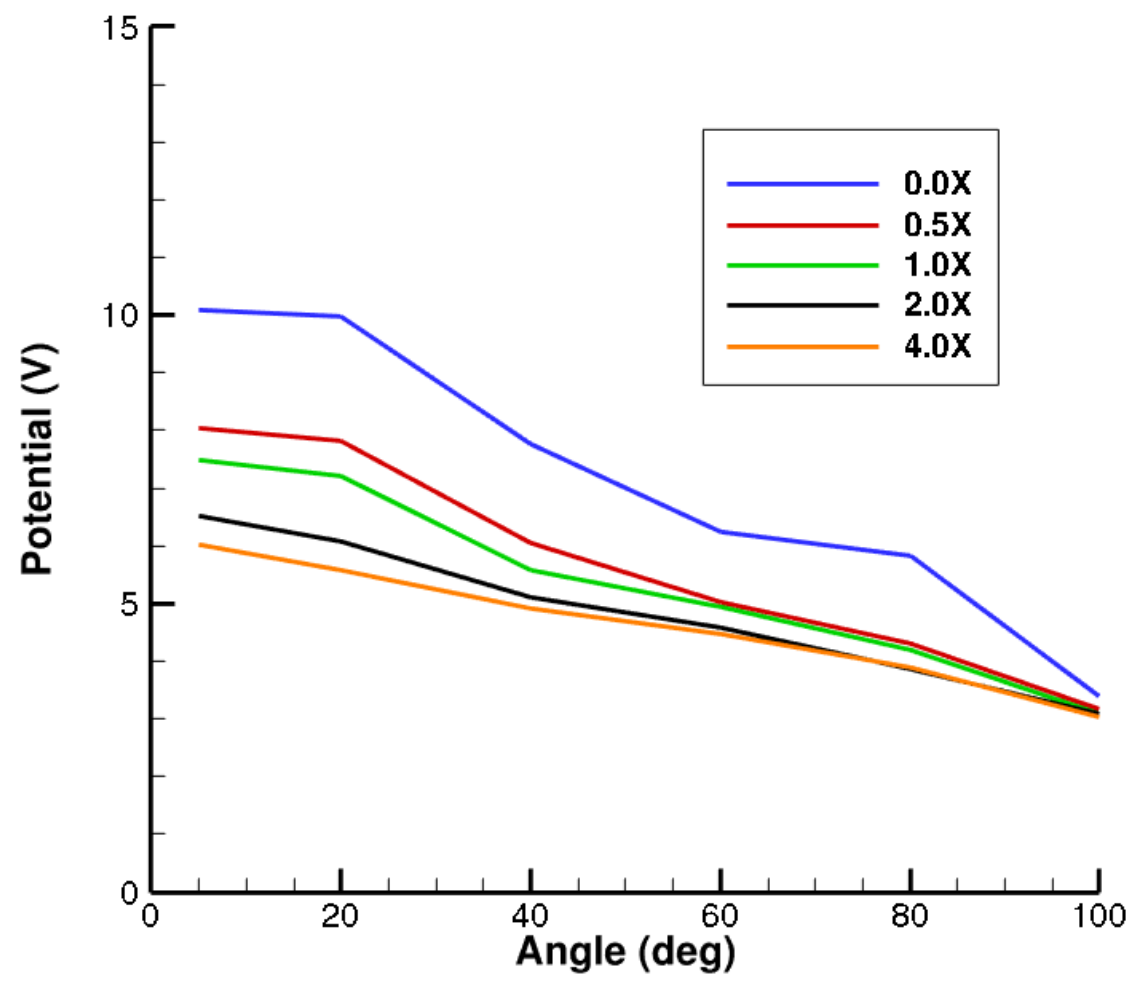

Figure 4. Angular dependence of plasma potential, $1 \mathrm{~m}$ from thruster exit, at various background pressures. 


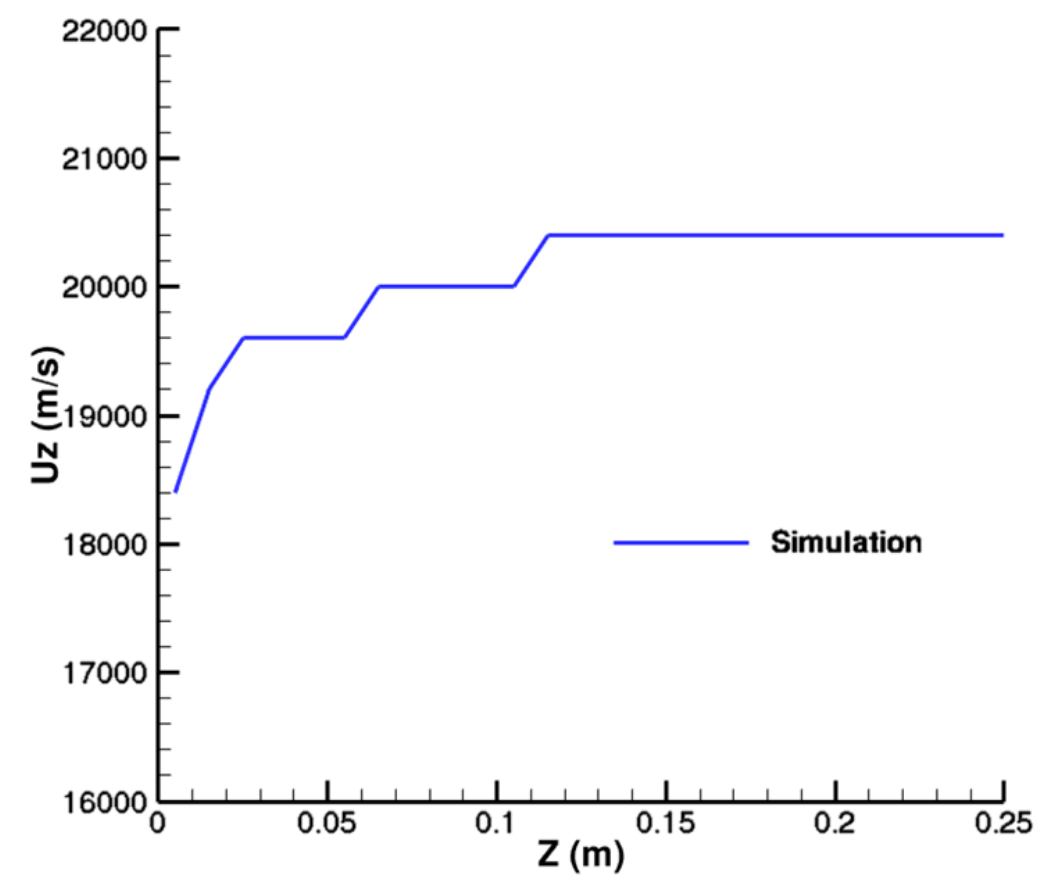

Figure 5. Calculated peak axial velocity for $\mathrm{Xe}^{+}$beam ions from exit to $25-\mathrm{cm}$ downstream, for $1 \mathrm{X}$ case.

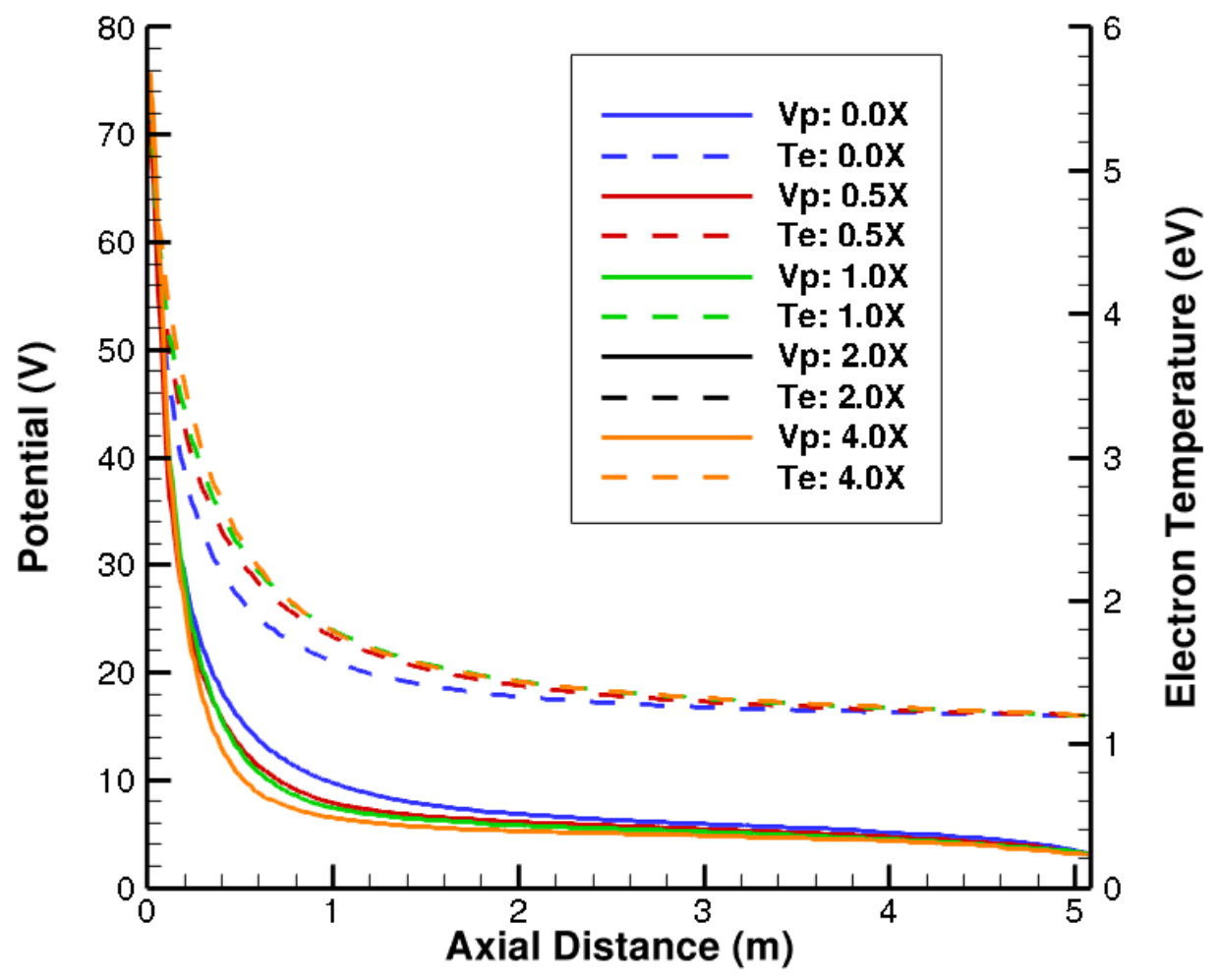

Figure 6. Plasma potential and electron temperature axial profiles. 

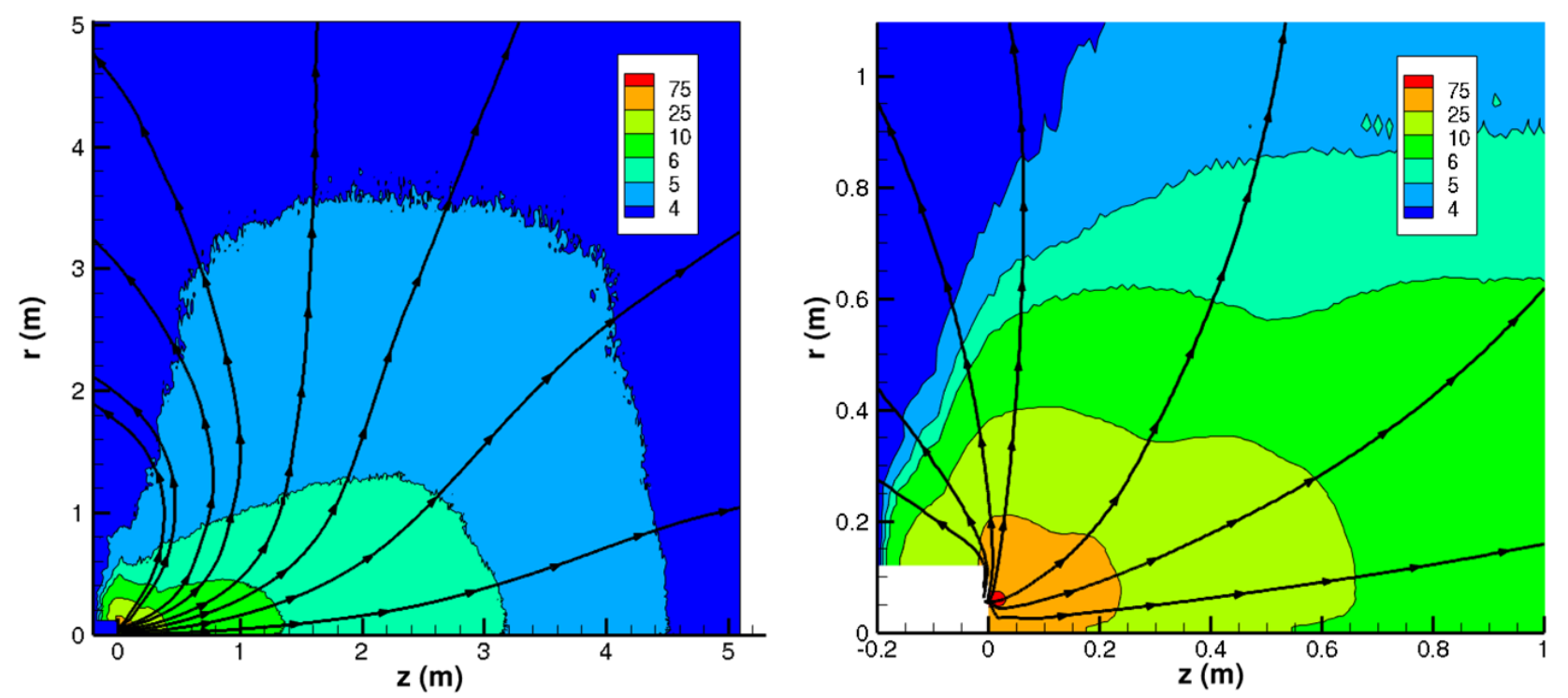

Figure 7. Computed ion trajectories and contours of plasma potential for $1 \mathrm{X}$ case. The right plot is a zoomed version of the left plot.

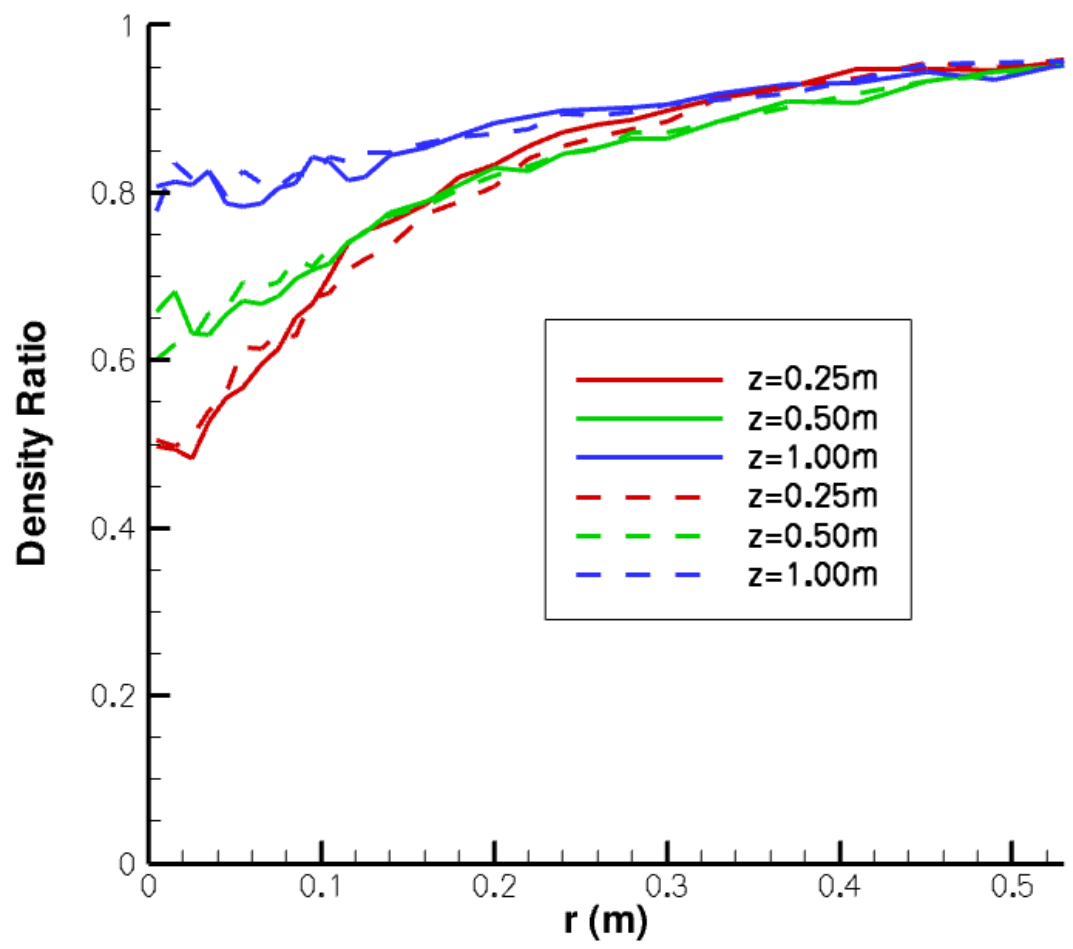

Figure 8. Contours of background gas density ratio with and without the inclusion of neutrals flowing out of the thruster. 


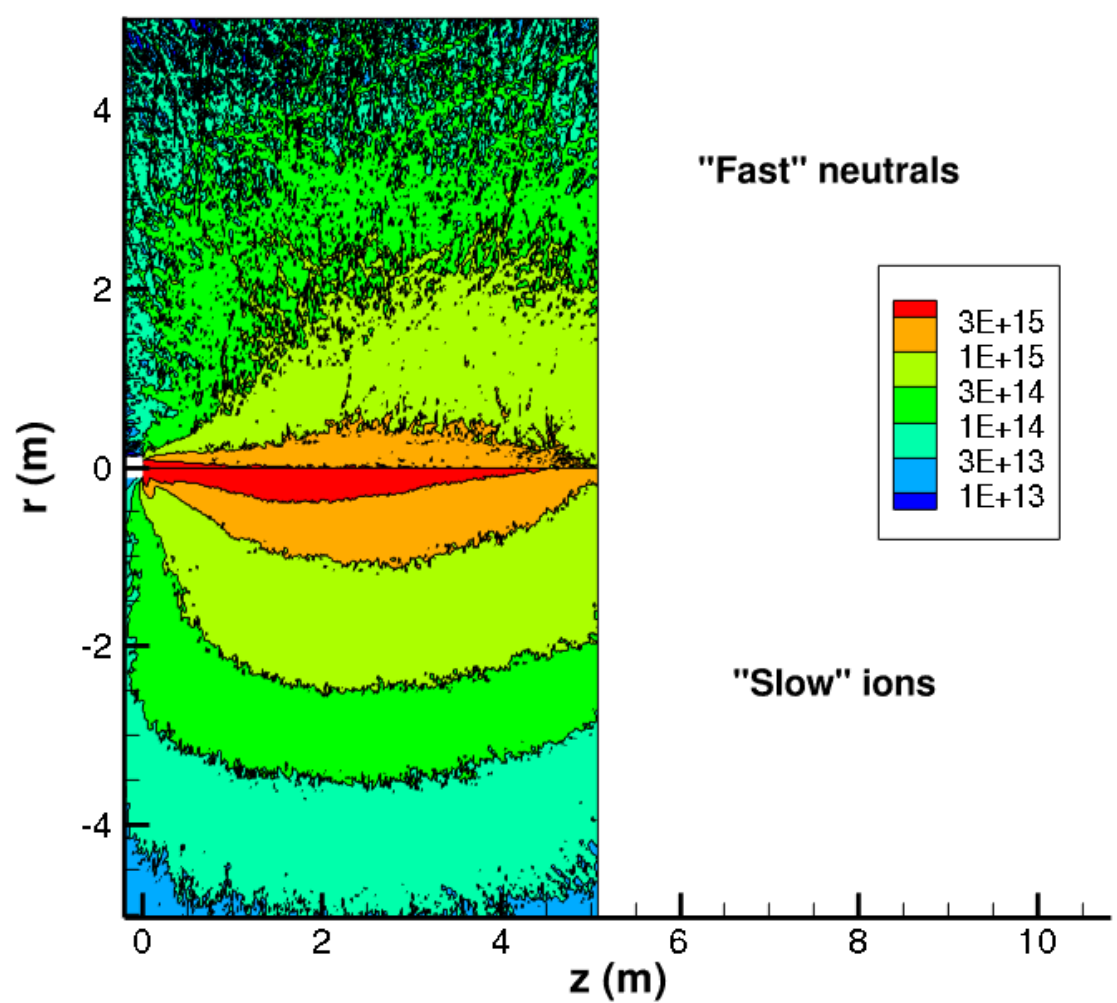

Figure 9. Number density contours $\left(\mathrm{m}^{-3}\right)$ for fast neutrals and slow ions in the plume.

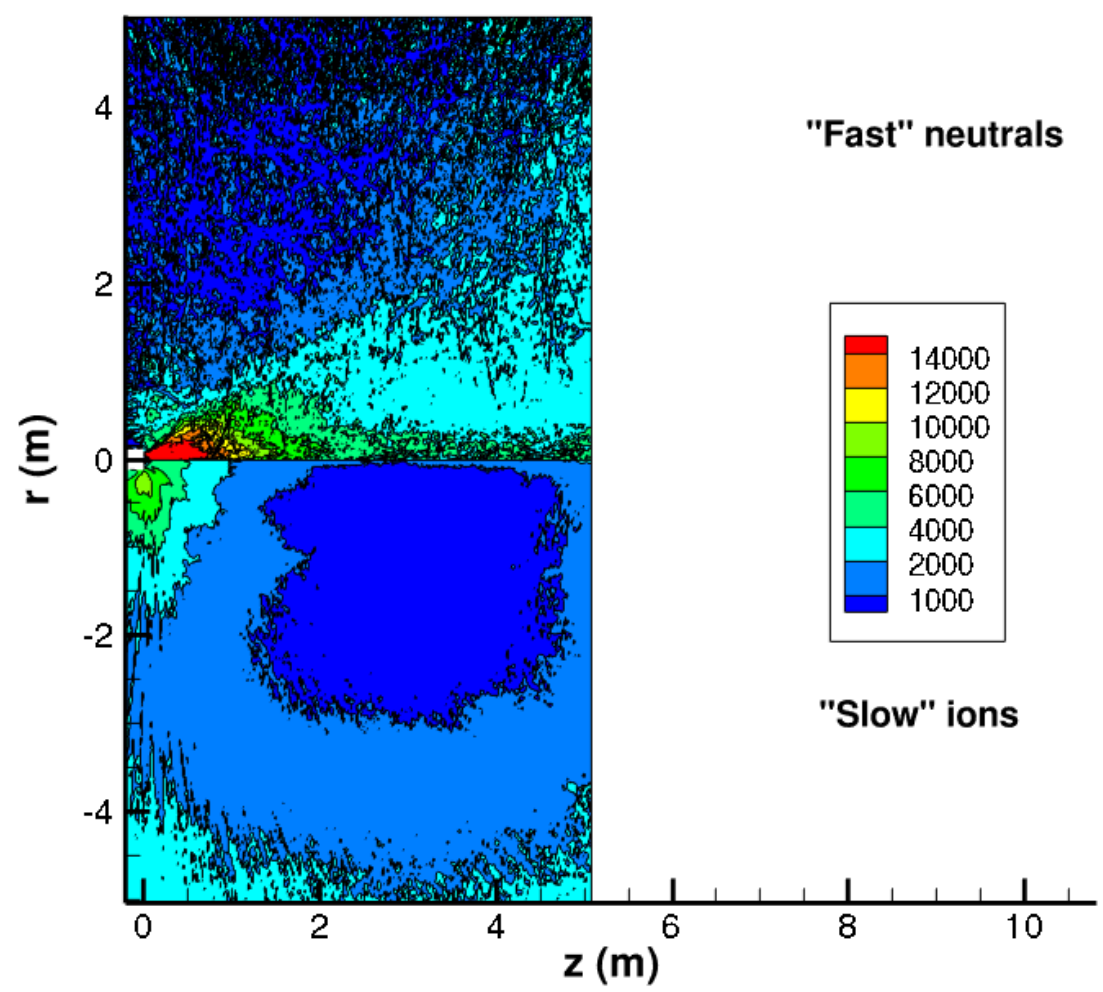

Figure 10. Velocity contours for fast neutral and slow ion charge exchange products. 
The angular dependences of electron temperature and density at 1-m distance, also for selected background pressures from 1X to 4X, are plotted in Figures 2 and 3, respectively. The electron temperature shows little angular dependence below 40 degrees, with modest decline at higher angles. Its dependence on background pressure is quite small, which is significantly reduced relative to no-field results, and of opposite sign with respect to no-field and experimental results. ${ }^{13}$ Electron (plasma) density variation with background pressure, as shown in Figure 3, is strong. Electron number density increases monotonically with xenon background density, with an order of magnitude or more electron density at $4 \mathrm{X}$ pressure vs $0 \mathrm{X}$, for angles equal to or greater than 30 degrees. In the small-angle core of the plume, the computed electron density still depends very substantially on background xenon pressure; for the no-field case dependence was much less. Differences between computed and experimental electron temperature and density curves at $1 \mathrm{~m}$ are not insignificant.

The simulated angular plasma potential curves exhibit both angular and pressure dependence, as shown in Figure 4. The angular dependence is reduced at high pressure, and the magnitude of the potential decreases with increasing pressure. These results are validated well by experimental measurements ${ }^{13}$, and are critical for the present study.

Ion velocity at the exit plane and just downstream can be related directly to the local plasma potential and its delta with respect to where the ion was born. Comparison of the simulation result, shown in Figure 5, with representative laser induced fluorescence data indicates reasonable agreement. $\mathrm{Xe}^{+}$axial velocity of $18 \mathrm{~km} / \mathrm{s}$ at the exit corresponds to about $221 \mathrm{eV}$ of kinetic energy, so the plasma potential there would be $79 \mathrm{~V}$ if the ions were born at $300 \mathrm{~V}$ (not realistic due to combined effects of anode fall and the negative floating potential of the cathode; anode to cathode $\Delta \mathrm{V}$ is fixed at $300 \mathrm{~V}$ ) and $49 \mathrm{~V}$ for ions born at $270 \mathrm{~V}$, a more reasonable value. Plasma potential and electron temperature axial profiles are given in Figure 6. Plasma potential drops quickly in the near field region, from $22 \mathrm{~V}$ at $30 \mathrm{~cm}$ to $10 \mathrm{~V}$ at $100 \mathrm{~cm}$, compared to the previous no-field result of $12 \mathrm{~V}$ at $30 \mathrm{~cm}$ and $6 \mathrm{~V}$ at $100 \mathrm{~cm}$. Plasma potential decreases relatively slowly at larger distances. Electron temperature also drops quickly at first and stabilizes at about the same distance, eventually becoming fairly insensitive to increasing distance and angular coordinate. It is high enough near the exit plane to cause non-negligible electron impact ionization of neutrals.

Plasma potential contours and slow ion trajectories are plotted in Figure 7 for the $1 \mathrm{X}$ case. A radial velocity component tends to be favored over axial, and the axial component takes both positive and negative values. Negative values will decrease the integral sum of axial momentum flux.

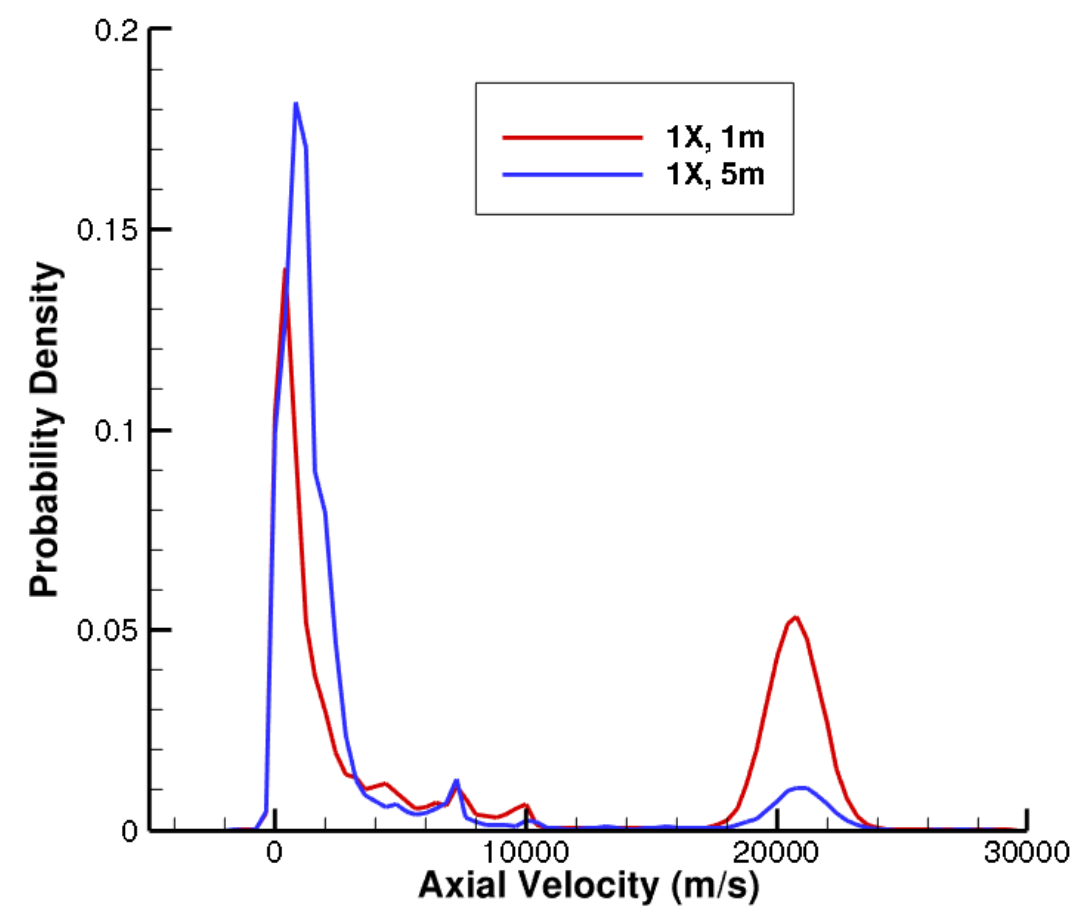

Figure 11. Axial velocity distribution function for ions at 1 and $5 \mathrm{~m}$ distances, with $1 \mathrm{X}$ facility pressure, on linear scale. 
As confirmation of the analytical model for radial profiles of neutral xenon in the plume due to diffusion of background gas, ${ }^{3}$ simulated radial profiles obtained from the present calculations show similar behavior. The ratio of neutral density along radial cuts through the plume, relative to chamber wall density, is plotted in Figure 8 . There is no significant difference at any axial distance, with and without inclusion of thruster source flow. As expected, a minimum exists on the plume axis that becomes progressively more shallow with increasing axial distance. At 25$\mathrm{cm}$ the centerline density is about $50 \%$ of the wall density. The dip would be more pronounced nearer the exit plane.
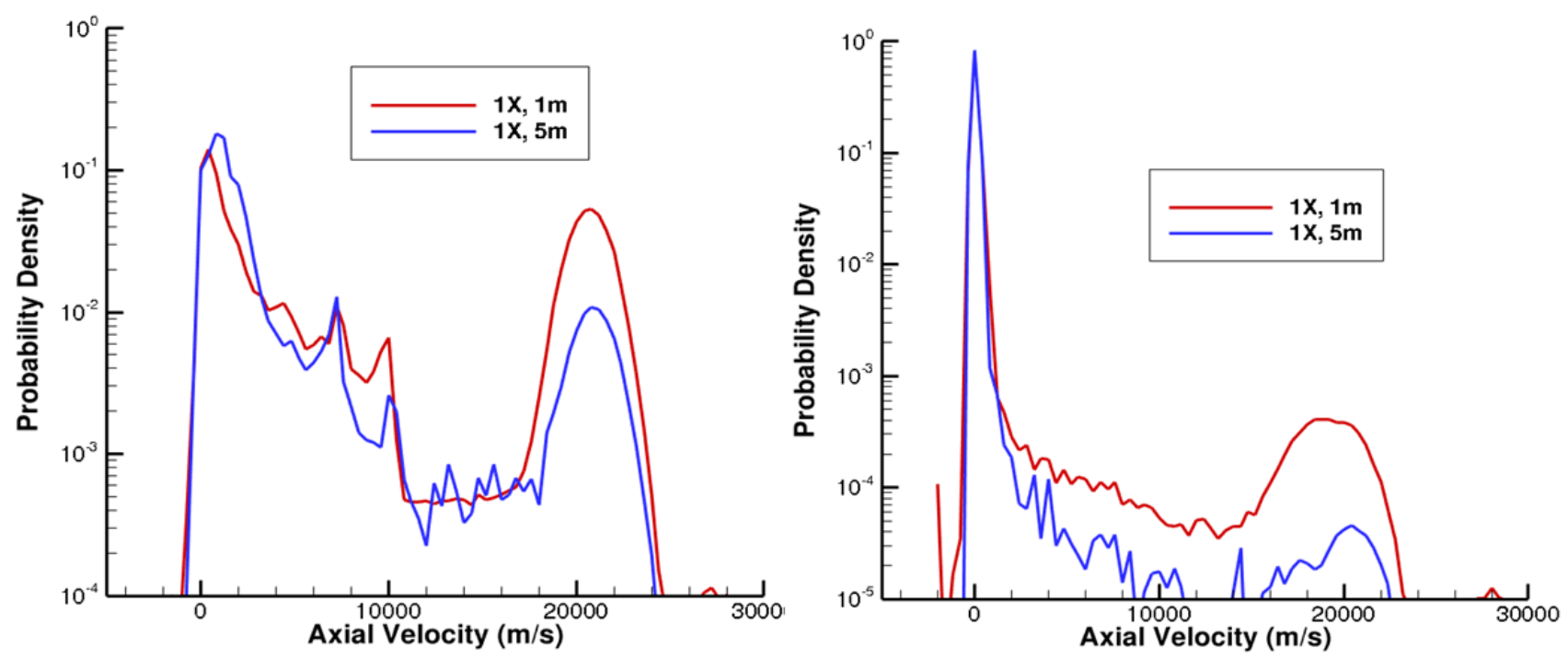

Figure 12. Axial VDF for ions (left) and neutrals (right), $1 \mathrm{X}$ pressure at 1 and $5 \mathrm{~m}$.
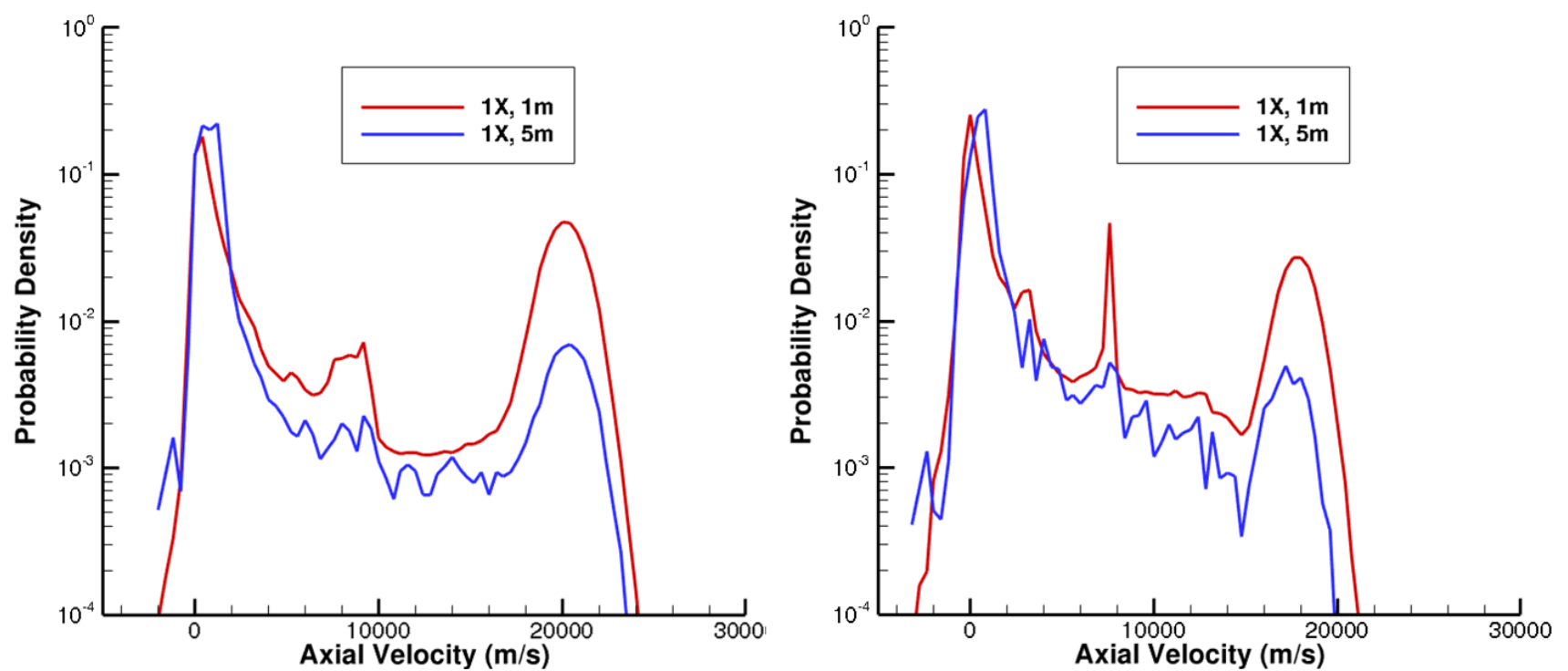

Figure 13. Axial VDF for ions 20 degrees off-axis (left) and 40 degrees off-axis (right).

Plume neutrals, as well as ions, can be differentiated by their velocity characteristics, since "fast" neutrals and "slow" ions are efficiently produced in pairs by charge exchange, and background neutrals are themselves moving very slowly in comparison. Fast neutral and slow ion number density contours are displayed in Figure 9, where it is apparent that the slow ions have significantly higher density - presumably due to their relatively low velocity. While the spatial distributions differ, both species have highest density near the exit plane of the thruster, decreasing in both radial and axial directions. Roughly speaking, one to two orders of magnitude drop in density occurs in axial and radial directions, respectively, over a range of several meters. The velocity contours for these species are plotted 
in Figure 10, showing that the fast neutrals start out with much higher velocity than slow ions, near the exit plane. In the mid-field region of 1-2 meters from the thruster, it appears that the slow ions go through a minimum average velocity region. This may result from high production rates of both slow and elevated velocity ions near the exit plane, combined with significant conversion of these into the slow category as they migrate downstream. High average velocity and density is produced near the exit plane, low velocity and medium density in the mid-field region, and low density with modestly higher velocity at greater distances. These far-field properties occur because few ions are born at large distance and ions coming from upstream have experienced a long acceleration. Mid-field properties occur because most ions there were birthed at low potential relative to the near-field and accelerate moderately in both axial and radial directions due to modest electric fields. In the near-field region production is high and electric fields are relatively large. Fast neutrals formed near the exit plane typically will not retain their high velocity indefinitely as they travel away, due to collisions with background neutrals. With a mean free path that is on the order of several meters, multiple collisions are possible within the computational domain.

Outcomes of the various plume ion interactions span a wide spectrum. Because of the broad initial velocity distribution of beam ions, emitted over a broad angular distribution with varying plasma potential, electric field and neutral density throughout the plume, the tracking of individual nuclei on their journey and classifying the various particles with their respective velocity and density distributions is complex. Here we focus on the velocity distribution functions for whole classes of particles: ions and neutrals. The total number of ions and neutrals in the plume varies little, since plume ionization is modest and we neglect the relatively slow process of recombination. It is instructive to study how the velocity distribution function varies within the plume, and it is a necessary quantity for calculating the total momentum crossing an arbitrary surface. By simulating the conversion of fast ions to slower ions and their subsequent acceleratation by local fields throughout the plume, and summing the momentum, thrust augmentation associated with plume processes can be estimated.

An axial VDF for ions at $1 \mathrm{~m}$ and $5 \mathrm{~m}$ distances for the $1 \mathrm{X}$ pressure case is plotted on a linear scale in Figure 11. Ions are emitted from the thruster with velocity approaching $20 \mathrm{~km} / \mathrm{s}$, with a broad peak in that portion of the velocity spectrum due to the distribution of birth potentials. Ions with low velocity are primarily formed via ionneutral CEX interactions and electron impact ionization. These low energy ions are apparent in the VDF, with a large peak at very low velocity relative to beam ion velocity. They are likely to be formed mainly by elastic CEX reactions, whereas ions produced via electron impact will peak at higher velocity, having at least several $\mathrm{eV}$ of energy. Axial velocity of $2 \mathrm{~km} / \mathrm{s}$ is distinct from the prominent low velocity peak in the VDF, but corresponds to less than $3 \mathrm{eV}$ axial energy. The small peaks at about 7 and $10 \mathrm{~km} / \mathrm{s}$ are probably formed via electron impact ionization very near the thruster exit plane, where plasma potentials are $50-80 \mathrm{~V}$. There is little difference between their peak positions at 1 and $5 \mathrm{~m}$, because - like beam ions - an additional $5 \mathrm{eV}$ of energy doesn't add very much to their velocity. Medium energy ions are also formed through scattering processes, with smoothly varying probability that results in broad distribution with respect to velocity and low probability per velocity increment compared to the ion groups at the very low and very high ends of the spectrum. Comparison between the $1 \mathrm{~m}$ and $5 \mathrm{~m}$ distributions shows that beam ions are efficiently converted to low energy ions as they propagate away from the thruster. The low energy population has $\sim 1 \mathrm{~km} / \mathrm{s}$ higher axial velocity at $5 \mathrm{~m}$ than at $1 \mathrm{~m}$, and a slightly broadened velocity distribution, indicating that ions produced upstream have accelerated along their path to the $5 \mathrm{~m}$ point. The low energy population at $1 \mathrm{~m}$ has a tail on the high velocity side, which seems to be missing at $5 \mathrm{~m}$. Although barely noticeable, the high energy peak at $5 \mathrm{~m}$ has its maximum shifted to slightly higher velocity than the corresponding $1 \mathrm{~m}$ peak, for the same reason as the low energy peak. Assuming the same energy gain applies to these cases, the velocity increment for the high energy peak would be much less, as observed.

Ion and neutral axial VDFs are plotted at 1 and $5 \mathrm{~m}$ for the $1 \mathrm{X}$ pressure case in Figure 12. The ion VDF is identical to the plot given in Figure 11, except done on a log scale. It is immediately apparent in the right panel that the neutral low energy peak greatly dominates over the high energy peak at both 1 and $5 \mathrm{~m}$. This occurs because of the abundance of thermal neutrals from the background, with other velocities produced only through a collisional interaction. Like the ion case, medium energy species are less abundant per unit energy than those with "beam" energies. The small medium energy peaks observed for the ion case are missing, as expected. At $5 \mathrm{~m}$ the probability density of the high energy peak is much less than at $1 \mathrm{~m}$, probably because most fast neutrals formed upstream have high angle trajectories and are quickly lost, while the on-axis production rate of fast neutrals is much lower at $5 \mathrm{~m}$ than at $1 \mathrm{~m}$. Because of plume expansion and beam ion losses, the current density at the $5 \mathrm{~m}$ location is far less.

Ion off-axis VDFs at $1 \mathrm{~m}$ and $5 \mathrm{~m}$ distance and $1 \mathrm{X}$ pressure are plotted in Figure 13 for $\theta=20$ (left) and 40 (right). Again, the low energy peak is noticeably accelerated from $1 \mathrm{~m}$ to $5 \mathrm{~m}$.

To evaluate momentum flux from the thruster and evaluate the way it changes with time, we draw an arc (circle) around the thruster and determine the flux across this boundary. The distance between thruster and boundary can be varied. Figure 14 shows the integrated axial momentum flux for beam ions and lower energy ions across the 
boundary as a function of radial distance. Beam ion momentum flux has very little dependence on background pressure, since they originate at fixed potential and "terminate" at $3 \mathrm{~V}$ fixed potential as a downstream boundary condition. Because of their high energy, trajectories are largely unaffected. The slow ion trajectories and velocity distributions are affected much more by the effects of background pressure, as can be seen in the Figure. The upswing near $5 \mathrm{~m}$ may be influenced by the boundary condition imposed there. The downswing in the mid-range under $2 \mathrm{X}$ and $4 \mathrm{X}$ conditions may be associated with unfavorable trajectories for accumulation of positive axial momentum flux.
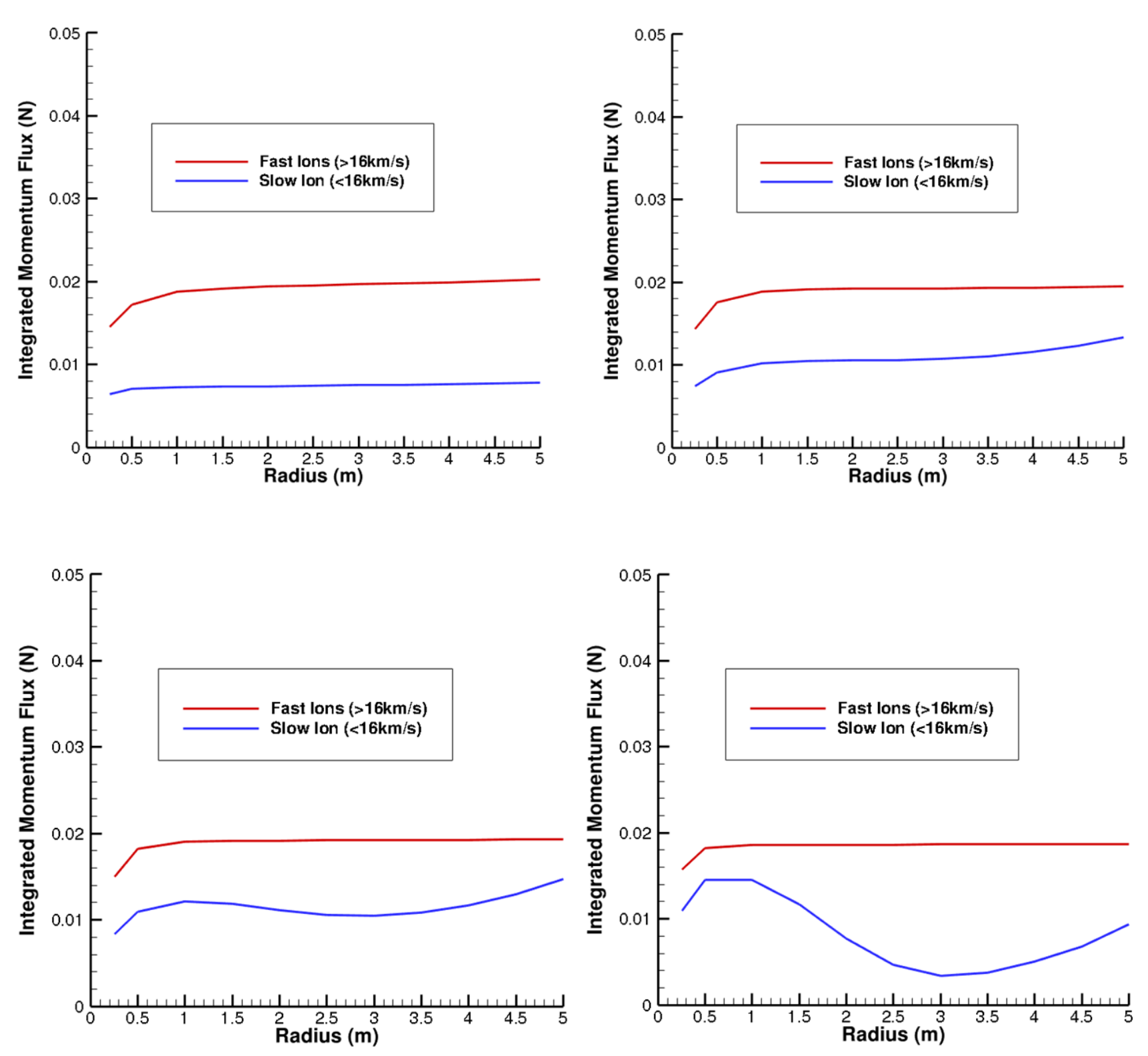

Figure 14. Integrated plume axial momentum flux for the indicated species, as a function of spherical boundary distance from the thruster, for $0 \mathrm{X}$ (top left), $1 \mathrm{X}$ (top right), $2 \mathrm{X}$ (bottom left) and $4 \mathrm{X}$ (bottom right) pressure conditions. 

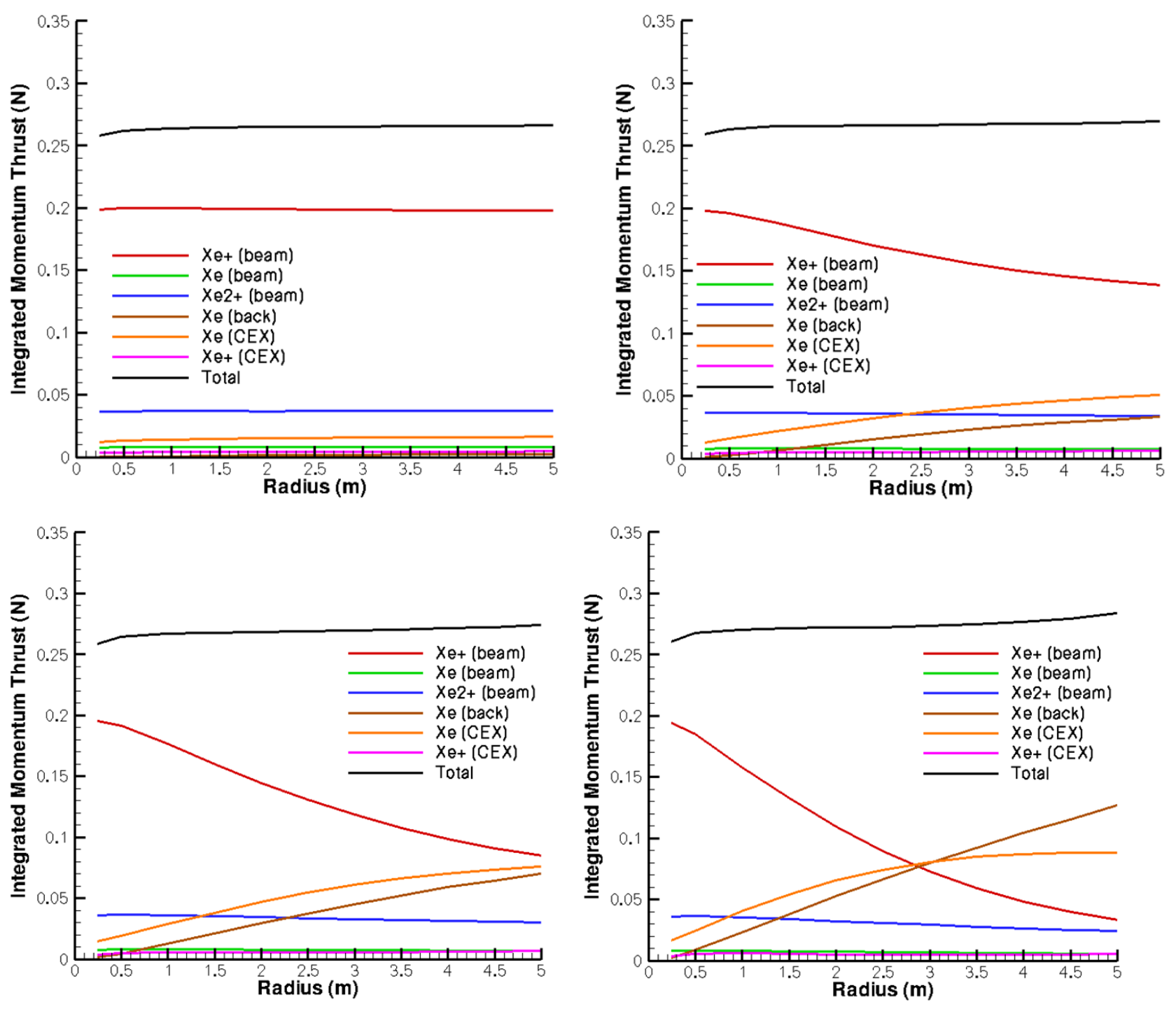

Figure 15. Integrated momentum flux per species as a function of distance from thruster, for $0 \mathrm{X}$ (top left), $0.5 \mathrm{X}$ (top right), $1 \mathrm{X}$ (bottom left) and $2 \mathrm{X}$ (bottom right) background pressures. $\mathrm{Xe}(\mathrm{CEX})=$ fast atoms; $\mathrm{Xe}+(\mathrm{CEX})=$ slow ions; Xe (beam) = neutrals emitted by thruster; Xe+ (beam) = singly charged ions emitted by thruster; Xe $($ back $)=$ background atoms; Xe2+ $($ beam $)=$ doubly charged ions emitted by thruster, and unlike Xe+ (beam) the profile includes both beam and CEX particles.

Species-specific momentum flux contributions for four different background pressures are plotted in Figure 15. In addition to the total thrust, as determined from the summed momentum flux crossing a thruster-centric spherical surface with 5-m radius, individual contributions are included in the plot. In the Figure, Xe (back) denotes thrust contribution from background atoms that underwent beam-ion scattering without charge exchange, Xe (CEX) denotes atoms that were beam ions before undergoing a CEX event and becoming neutralized, and Xe+ (CEX) denotes ions produced by CEX collisions involving neutrals from the source and background ensemble. If these ions undergo another CEX collision they will then be counted as beam species - about one-tenth do this.

The summed contributions of ions and neutrals, as a function of background pressure, are given in Figure 16. It is apparent that ion momentum is being transferred into neutral momentum, with efficiency that depends nonlinearly on background xenon density. The $1 \mathrm{X}$ case corresponds to roughly $50 \%$ momentum transfer, with neutrals the dominant momentum carrier for the $2 \mathrm{X}$ case. Average ion velocity is decreasing as the relative population of slow CEX ions increases and the average neutral velocity rises. 

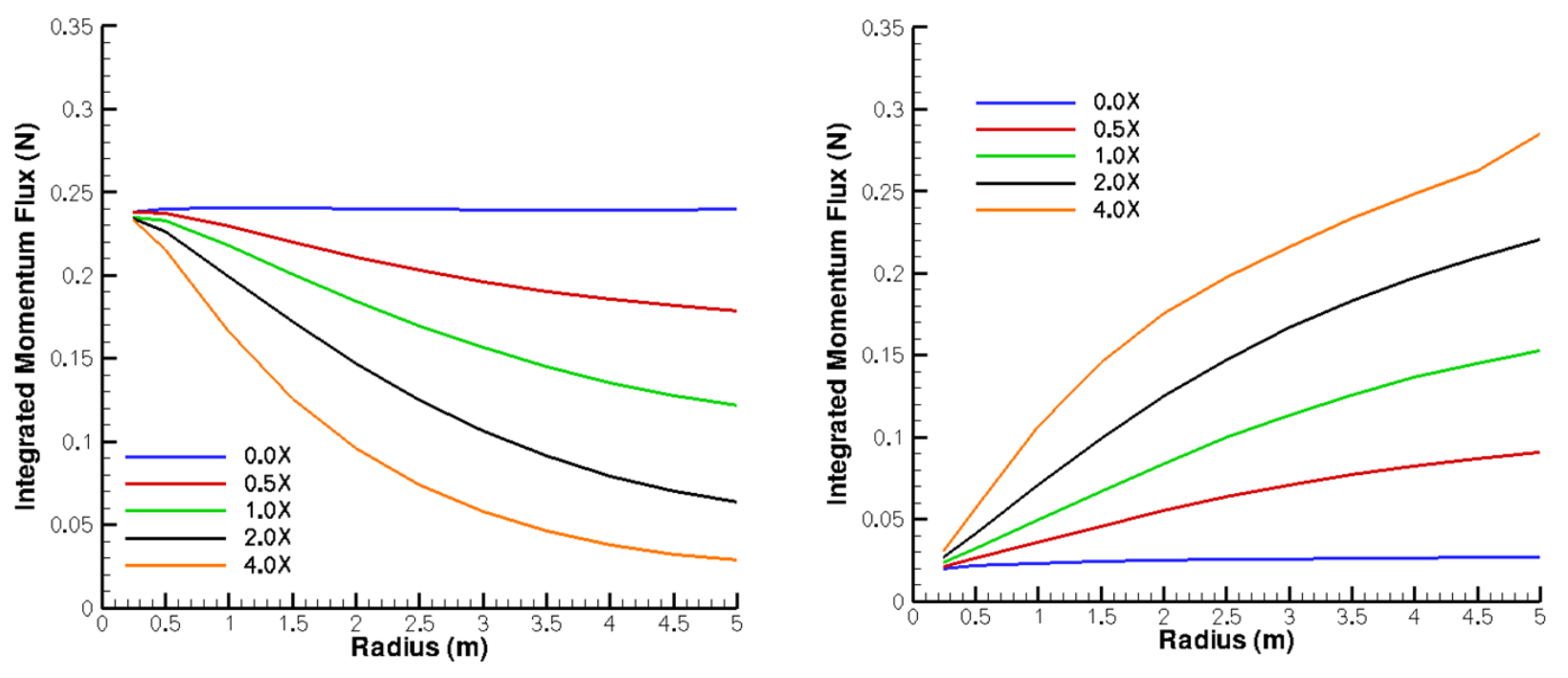

Figure 15. Integrated momentum flux per species for ion (left) and neutral (right) ensembles as a function of integrating sphere radius and background pressure.

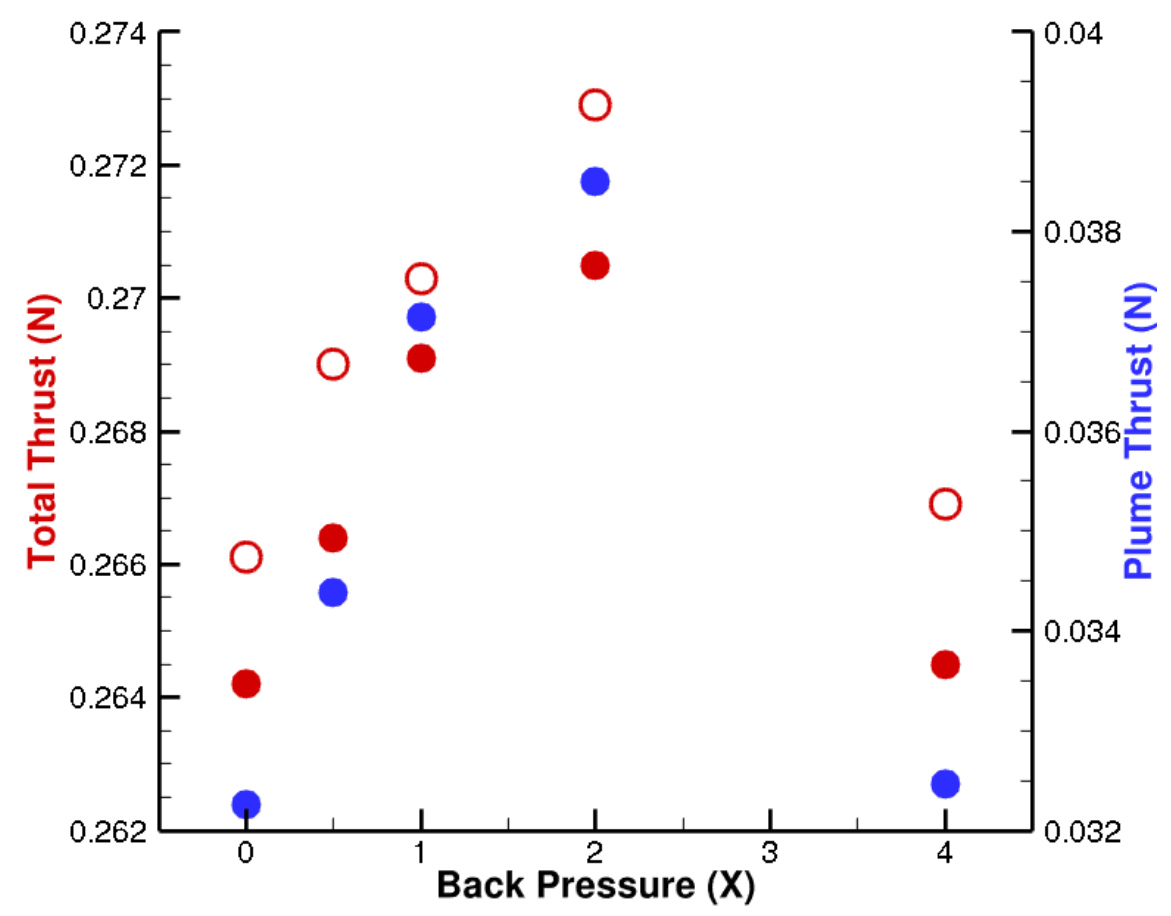

Figure 16. Integrated momentum flux crossing the spherical surface at 5-m distance, and the plume-only contribution. 


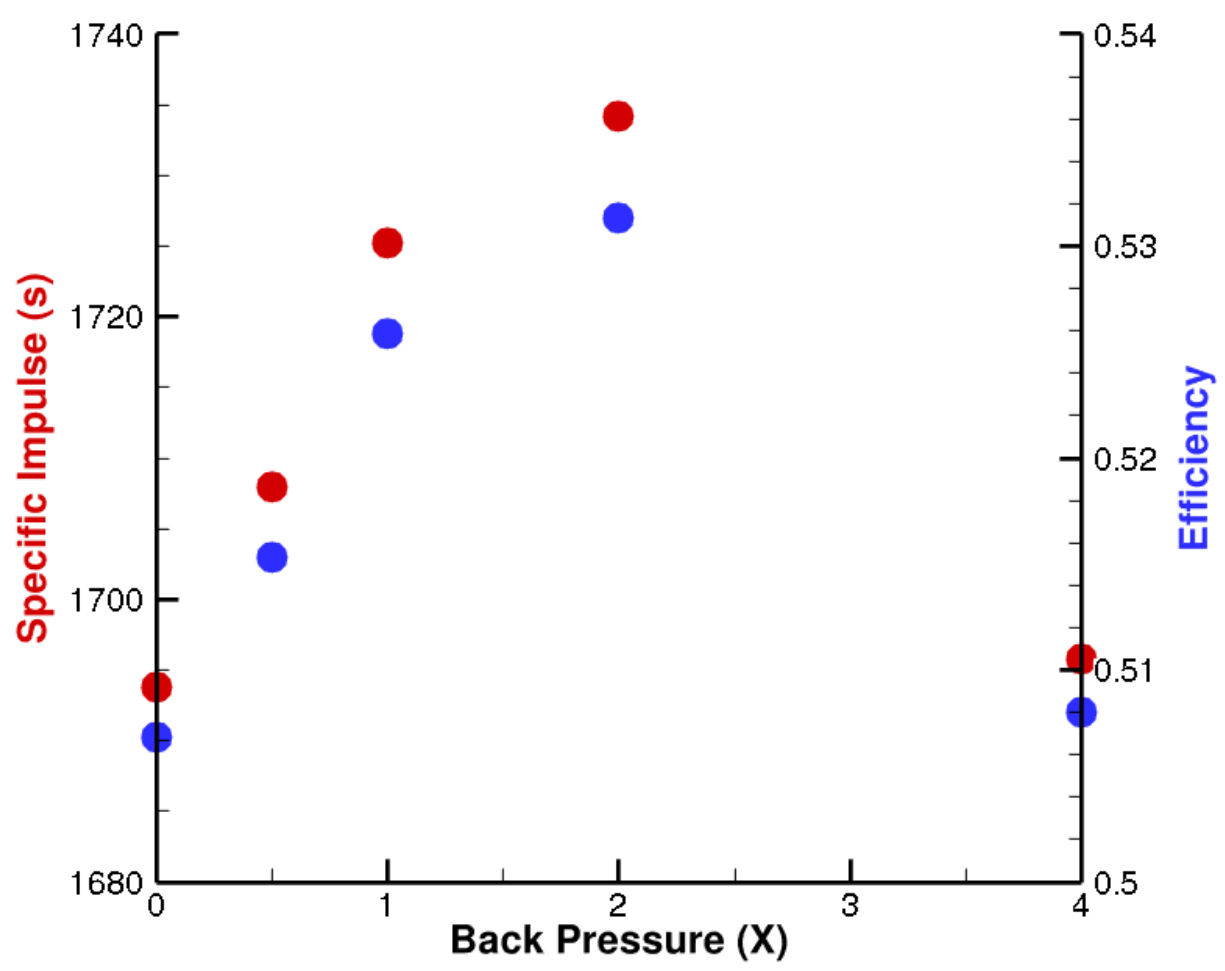

Figure 17. Specific impulse and efficiency as a function of the background pressure factor.

The only acceleration process in the plume simulation is due to electrostatic forces acting on the ions. By summing the associated velocity changes for every ion particle in the simulation, and averaging over time, the total momentum imparted to the plasma can be evaluated directly. Using this approach, the integrated momentum flux crossing the surface of a sphere of 5-m radius, centered on the thruster, is plotted in Figure 17 as a function of the xenon back pressure factor. Here we label the vertical axis "thrust", because we consider the computed integral momentum flux at the $5 \mathrm{~m}$ arc to be an approximate prediction for thrust stand measurements. The result is similar to the 1-D analytical model result in dependence on background pressure for $0 \mathrm{X}-2 \mathrm{X}$, but with lower amplitude. As already discussed, ion trajectories and their effects are more appropriately treated in the present case, which accounts for both radial and backward streaming ions. Back-streaming accounts for a significant reduction of the integrated axial momentum flux, particularly at the higher pressures, and may explain the fall-off from $2 \mathrm{X}$ to $4 \mathrm{X}$. The magnitude of thrust augmentation over the $0 \mathrm{X}$ to $2 \mathrm{X}$ range is about $3 \%$ for the numerical model, about one-third of the variation found by the analytical model. Zero referencing the potential increases the integrated momentum flux in the numerical simulation by approximately the same amount for all background pressures, and has little effect on the $0 \mathrm{X}-2 \mathrm{X}$ augmentation. This result is also plotted in Figure 17 (open circles).

The analytical model made several approximations that were not necessary for the more sophisticated treatment used here. These include a single-collision assumption, uniform current density across radial cuts, neglect of $\mathrm{Xe}^{2+}$ and its reactions, neglect of collisions where there is non-negligible momentum transfer between nuclei, and assumption of straight-line axial or near-axial trajectories. The last assumption is probably the most important, and could readily account for a doubling of estimated momentum flux increases with background pressure. The analytical model was useful for the prediction that momentum flux would vary with background pressure, but not a very quantitative treatment. Given the various approximations involved, the agreement between models seems acceptable.

The specific impulse and efficiency, obtained from the standard definitions $I_{s p}=T / m g$ and $\eta_{T}=T^{2} / 2 \dot{m} P_{i n}$, respectively, where $\dot{m}$ is mass flow rate, $T$ is thrust and $P_{i n}$ is input power, are plotted in Figure 18. Both increase initially with xenon background pressure, as has been observed in a number of past studies with various thrusters. ${ }^{1,14}$ The fractional efficiency variation is about twice the thrust variation; it is roughly $5 \%$ lower for $0 \mathrm{X}$ than $2 \mathrm{X}^{1,15}$ 
As mentioned, slow ion production from electron impact ionization in the plume was included in the simulations. Although its momentum contribution was found to be smaller than the sum of CEX and scattering components at $1 \mathrm{X}$ pressure, it is far from negligible, and a sensitive function of the electron temperature.

We have made no attempt to include background gas ingestion into the thruster itself, as the goal was to evaluate the effects of background gas diffusion into the plume only. More importantly, transient effects (e.g. breathing mode oscillations) were left out, as they are difficult to include. The current model provides a strong prediction that background xenon density modifies plume momentum flux. Further refinements would be required before it can hope to provide quantitative predictions. In addition, before it can be employed to evaluate a given thruster system it must be "calibrated" for that thruster.

\section{Conclusions}

Numerical simulation techniques were used to track the evolution of species-specific and total plume momentum throughout a Hall thruster plume. Detailed calculations were performed to determine velocity and density distribution functions for the population of ions and neutrals within the plume volume. A DSMC approach was used to model the collisions of the ions and neutrals, with PIC modeling of ion transport in the plume electric field. All significant particle interactions were included in the model, and specific emphasis was placed on the dependence of velocity distribution functions and total plume momentum on the density of background xenon gas. To obtain the results, the coordinate-dependent influence of plume parameters such as ion flux density, neutral density, electron temperature and plasma potential was included in the calculations. Results confirm that significant plume interactions occur, with initial ion beam momentum becoming substantially distributed between ion and neutral populations as the ion beam is attenuated, and increasing total momentum flux across a thruster-centric hemispherical surface at increasing downstream distance. Velocity distribution functions continue to change significantly with propagation distance beyond $1 \mathrm{~m}$ and exhibit angular dependence. Momentum augmentation is primarily due to collision-based reduction of average ion momentum as average neutral momentum increases, and the on-going acceleration of ion populations in the local electric field. The increase of plume momentum with rising background gas density is less than predicted in an earlier 1-D analytical study, which did not consider the effect of motion along the radial coordinate. The main difference between the current study and our preliminary report, is the inclusion of magnetic field effects. Time dependent effects are still absent from the model.

Plume momentum contributions from ions as a function of their birth location are most important in the thruster near-field region, due to ion beam attenuation and decreasing local plasma potential at birth. In addition, the axial directivity seems to vary considerably with distance from thruster and background pressure. As a result, large facility dimensions are not required to realize similar results. The plasma potential distribution is a critical input to the calculations, affecting both energy and trajectory. Equating plume momentum augmentation with increased thrust level, the specific impulse and efficiency increases with background pressure. The improved performance results from plume ingestion of additional propellant, as opposed to ingestion across the exit plane, and conversion to slow ions that gain more momentum in the local electric field than fast ions. Average ion momentum decreases as average neutral momentum increases. Ground-test performance tends to increase with thruster power, due to this mechanism, other factors remaining constant.

Experimental measurements of near-field potential, electron temperature, and electron/neutral density maps, including transient behavior, for a variety of thrusters, would be helpful in further elucidating thruster physics and refining the model presented here.

\section{Acknowledgments}

This work was supported under The Aerospace Corporation's Independent Research and Development Program, managed by Rosy Atmadja and Paul Rousseau. The authors thank Jim Pollard for technical discussion.

\section{References}

1. Byers, D. and Dankanich, J.W., "A Review of Facility Effects on Hall Effect Thrusters," IEPC Paper 2009-076, $31^{\text {st }}$ International Electric Propulsion Conference, Ann Arbor, Sept 2009.

2. Katz, I., et al, "A Hall Effect Thruster Plume Model Including Large-Angle Elastic Scattering," AIAA Paper 2001-3355, $37^{\text {th }}$ Joint Propulsion Conference, July 2001, Salt Lake City.

3. Crofton, M.W. and Pollard, J.E., "Thrust Augmentation by Charge Exchange," AIAA Paper 2013-4131, 49 Joint Propulsion Conference, San Jose, July 2013. 
4. Garrigues, L., Mazouffre, S., and Bourgeois, "Computed versus Measured Ion Velocity Distribution Functions in a Hall Effect Thruster," Journal of Applied Physics 111 (2012), p. 113301.

5. Mazouffre, S., Bourgeois, G., Garrigues, L., and Pawelec, E., "A Comprehensive Study on the Atom Flow in the Cross-Field Discharge of a Hall Thruster," Journal of Physics D: Applied Physics, Vol. 44, 2011, p. 105203.

6. Boyd, I.D., "Review of Hall Thruster Plume Modeling," Journal of Spacecraft and Rockets, Vol. 38, 2001, pp. 381-387.

7. Boyd, I.D. and Dressler, R.A., "Far Field Modeling of the Plume of a Hall Thruster," Journal of Applied Physics, Vol. 92, 2002, pp. 1764-1774.

8. Bird, G.A., Molecular Gas Dynamics and the Direct Simulation of Gas Flows, Oxford University Press, Oxford, 1994.

9. Birdsall, C.K. and Langdon, A.B., Plasma Physics Via Computer Simulation, Adam Hilger Press, 1991.

10. Boyd, I.D. and Yim, J.T., "Modeling of the Near Field Plume of a Hall Thruster," Journal of Applied Physics, Vol. 95, 2004, pp. 4575-4584.

11. Mitchner, M. and Kruger, C.H., Partially Ionized Gases, Wiley Press, New York, 1973.

12. Choi, Y., Boyd, I.D., and Keidar, M., "Effect of a Magnetic Field in Simulating the Plume of an Anode Layer Hall Thruster," Journal of Applied Physics, Vol. 105, Article 013303, 2009.

13. Diamant, K.D., unpublished data.

14.Fossum, E.C. and King, L.B., "Effects of Neutral Density on Electron Temperature and Mobility in a Crossedfield Trap," AIAA Paper 2008-5190, 44 ${ }^{\text {th }}$ Joint Propulsion Conference, Hartford, July 2008.

15.Walker, M.L.R. and Gallimore, A.D., "Performance Characteristics of a Cluster of 5-kW Laboratory Hall Thrusters," Journal of Propulsion and Power, Vol. 23, 2007, pp. 35-43. 